\section{Início da vida sexual na adolescência e relações de gênero: um estudo transversal em São Paulo, Brasil, 2002}

\author{
Sexual debut in adolescence and gender relations: \\ a cross-sectional study in São Paulo, Brazil, 2002
}

\author{
1 Escola de Enfermagem, \\ Universidade de São Paulo, \\ São Paulo, Brasil. \\ 2 Faculdade de Saúde \\ Pública, Universidade \\ de São Paulo, \\ São Paulo, Brasil. \\ Correspondência \\ A. L. V. Borges \\ Departamento de \\ Enfermagem em Saúde \\ Coletiva, Escola de \\ Enfermagem, \\ Universidade de São Paulo. \\ Rua Dr. Enéas de Carvalho \\ Aguiar 419, São Paulo, SP \\ 05403-000, Brasil. \\ alvilela@usp.br
}

\begin{abstract}
This cross-sectional study aimed to assess gender differences in adolescents in relation to onset of sexual activity. A total of 406 15-19-yearold adolescents from a family health unit in the city of São Paulo, Brazil, were interviewed from May to December 2002. Nearly half had already had their first sexual intercourse, at a mean age of 15, independently of gender. Sexual debut was usually unplanned (72.7\%) and inside the home (86.1\%). An equal proportion (61.0\%) of males and females used some contraceptive method in their first sexual intercourse; however, more males had used a contraceptive method in their most recent intercourse. Having initiated their sexual activity in stable, affective relationships, females proved to be more vulnerable to STD/ AIDS, since they switched from condoms to the pill in their subsequent sexual activity. Male adolescents continued to use mostly condoms, probably because they were involved mainly in non-stable relationships.
\end{abstract}

Reproductive Medicine; Adolescent; Interpersonal Relations
Ana Luiza Vilela Borges 1,2

Néia Schor 2

\section{Introdução}

Homens e mulheres têm iniciado sua vida sexual, em grande parte, na adolescência e de formas um tanto diferenciadas. As práticas sexuais na juventude têm sido descritas como dinâmicas e em constantes transformações, sendo que seus perfis podem acarretar impacto importante na vida reprodutiva dos jovens, como, por exemplo, o aumento das taxas de fecundidade na faixa etária dos 15 aos 19 anos de idade observado para o Brasil como um todo 1 e a magnitude da AIDS no perfil epidemiológico dos jovens brasileiros 2 .

A primeira relação sexual é considerada um marco na vida reprodutiva de qualquer indivíduo e tem ocorrido cada vez mais precocemente, fenômeno descrito em estudos mais recentes no Brasil. Ferraz \& Ferreira 3 relataram que, em 1986, apenas $14,0 \%$ dos jovens entre 15 e 19 anos tiveram relação sexual pré-marital, enquanto que, em 1996, esta proporção subiu para 30,0\%. Este aspecto foi também evidenciado em estudo realizado pelo Ministério da Saúde (MS) 4 , onde a idade média do início da vida sexual encontrada em 1984 foi 15,3 anos entre os homens de 16 a 19 anos de idade e 16 anos entre as mulheres da faixa etária. Já em 1998, a idade média verificada diminuiu para $14,5 \mathrm{e}$ 15,2 anos respectivamente.

Melo \& Yazaki 5 também constataram uma antecipação do início da vida sexual, especial- 
mente no grupo de mulheres de 15 a 19 anos. Em 1986, apenas 8,7\% destas jovens referiram ter tido relação sexual antes dos 15 anos de idade. Analisando os dados de 1996, foi verificado que este índice aumentou para $22,6 \%$. Cabe salientar que a antecipação da primeira relação sexual está presente nos diferentes estratos sociais, podendo ser admitida como uma tendência generalizada.

As questões de gênero têm se mostrado importantes na condução das escolhas reprodutivas de adolescentes, particularmente o momento da primeira relação sexual e o primeiro parceiro sexual. Na maioria das vezes, a iniciação sexual de jovens do sexo masculino ocorre mais precocemente que a de jovens do sexo feminino. No Brasil, a mediana da idade da primeira relação sexual foi de 19,5 anos para as mulheres e 16,7 anos para os homens em 1996 6. Em $1998,46,7 \%$ dos adolescentes do sexo masculino já haviam iniciado sua vida sexual antes dos 14 anos de idade, ao passo que a proporção de adolescentes do sexo feminino foi de $32,3 \% 4$. Pirotta 7 , ao estudar uma amostra representativa dos estudantes de graduação matriculados em uma universidade estadual paulista, encontrou uma mediana da idade da primeira relação sexual de 17 anos no grupo masculino e 18 anos no grupo feminino.

As explicações acerca das diferenças da idade do início da vida sexual entre homens e mulheres baseiam-se no fato de que as normas e expectativas sociais em relação à idade e circunstâncias adequadas para as primeiras práticas sexuais variam conforme o sexo ${ }^{8}$. Segundo Heilborn 9 (p. 401), "o regime das relações de gênero, prescrevendo condutas adequadas para homens e mulheres, intervém de maneira inequívoca nesse cenário de iniciação".

Torna-se necessário, então, aprofundar o conhecimento sobre as primeiras práticas sexuais de adolescentes e o encadeamento advindo do contexto escolhido para a iniciação da vida sexual de homens e mulheres jovens. Para tanto, o propósito deste estudo foi identificar as diferenças de gênero presentes no início da vida sexual de adolescentes de 15 a 19 anos de idade, inscritos em uma unidade básica de saúde da família do Município de São Paulo.

\section{Metodologia}

Trata-se de estudo transversal desenvolvido em uma amostra representativa de jovens entre 15 e 19 anos de idade, moradores da área adscrita de uma unidade de saúde da família da zona leste do Município de São Paulo, Bra- sil. Os sujeitos entrevistados foram selecionados por amostragem sistemática sem reposição, com base em uma listagem obtida por meio do Sistema de Informação da Atenção Básica (SIAB) e ordenada por micro-área e pelo número das famílias cadastradas no Programa Saúde da Família que tinham ao menos um adolescente entre 15 e 19 anos de idade. O tamanho amostral foi calculado baseando-se na precisão desejada para se estimar a porcentagem de adolescentes de 15 a 19 anos com vida sexual ativa. Tal proporção foi considerada igual a $60,0 \% 4$, com erro máximo em valor absoluto de 5,0\% e com nível de confiança de $95,0 \%$. Para se obter a estimativa final do tamanho da amostra, este valor foi ajustado usando-se um fator de correção para população finita. Ainda, considerando a possibilidade de perdas, foram acrescidos $30,0 \%$ para que não houvesse redução do tamanho da amostra.

A cada quatro famílias $(K=4)$, selecionouse um adolescente e, no caso de famílias com mais de um adolescente nesta faixa etária, um sorteio foi realizado. Para o início da seleção das famílias, sorteou-se uma entre as quatro primeiras na listagem.

Foram, então, entrevistados 406 jovens entre 15 e 19 anos de idade em seus próprios domicílios, por meio de um formulário estruturado, pré-testado e pré-codificado, entre junho e dezembro de 2002. Os jovens foram convidados a participar do estudo após uma explicação de seus objetivos e do conteúdo de suas questões. Foi enfatizado que as perguntas eram de cunho íntimo e que eles poderiam recusar a continuidade da entrevista a qualquer momento. Também foi assegurado que sua identidade seria mantida em sigilo. A entrevista foi realizada respeitando-se a privacidade do jovem e, mesmo tendo sido planejada para ser conduzida no próprio domicílio do adolescente, foi dada a opção para que ele escolhesse o local em que se sentisse mais confortável para responder as perguntas, como por exemplo, um cômodo específico da casa, quintal, casa de vizinhos/parentes, espaço público, centro de juventude e outros. Os consentimentos foram obtidos com os responsáveis pelo adolescente ou com ele próprio, desde que com idade igual ou superior a 18 anos. Esta pesquisa foi aprovada pela Comissão de Ética e Pesquisa da Faculdade de Saúde Pública, Universidade de São Paulo.

\section{Caracterização do grupo em estudo}

Entre os 406 jovens entrevistados, $222(54,7 \%)$ eram do sexo feminino e $184(45,3 \%)$ do sexo 
masculino. A idade média dos entrevistados foi $16,7$ anos ( $\mathrm{dp}=1,36)$. Os jovens classificaramse em sua maioria como negros $61,3 \%$ (249), sendo 171 de cor parda e 78 de cor preta. Os adolescentes que se referiram como brancos totalizaram $38,2 \%$ (155); um classificou-se como indígena e outro como amarelo. Esta proporção de adolescentes auto-classificados como negros é maior do que o percentual verificado no Brasil (45,3\%) e no Estado de São Paulo $(31,1 \%)$, de acordo com o Censo Demográfico de 2000 10. O aspecto raça/cor tem sido descrito como um importante marcador social, justamente porque a população jovem negra tem sido enfatizada como a mais excluída socialmente, pois, segundo Pinho et al. 11 (p. 279), "são eles que ocupam os piores níveis de escolaridade, apresentam as maiores dificuldades para ingresso no mercado de trabalho e, no momento em que nele se inserem, são aqueles que ocupam as mais desvalorizadas funções".

$\mathrm{O}$ fato de $25,3 \%$ destes jovens viverem em domicílios ocupados e 38,5\% morarem em domicílios cuja densidade de moradores por cômodo foi maior que um, o que revela condições habitacionais precárias, ratificou sua baixa inserção social.

A maioria dos adolescentes era estudante (75,4\% ou 306). O número expressivo de jovens ausentes do sistema educacional $(25,4 \%$ ou 100) merece ser destacado quando se considera que a maior parte dos estudos conduzidos com populações adolescentes, não apenas no campo da saúde reprodutiva, são realizados em escolas. Neste sentido, o fato deste presente estudo ter se constituído em uma pesquisa domiciliária foi fundamental para que se alcançasse uma amostra representativa de todos os adolescentes ali residentes, inclusive os que não estavam estudando, provavelmente os mais vulneráveis socialmente.

Entre os jovens que estavam ausentes do sistema educacional, apenas $38,3 \%$ já haviam concluído o ensino médio e outros $38,3 \%$ não chegaram sequer a terminar o ensino fundamental. É importante ressaltar que $63,0 \%$ dos ausentes do sistema escolar declaram-se completamente inativos. Entre os que estavam estudando, 26,5\% ainda cursavam o ensino fundamental, em um claro sinal de atraso escolar, ou seja, de defasagem na relação idade-série, visto que a faixa etária definida para o ensino fundamental é de 7 a 14 anos de idade 12. Desta forma, todos os indivíduos entrevistados, por encontrarem-se entre os 15 e 19 anos de idade, deveriam ter tido condições de concluir o ensino fundamental e ingressar no ensino médio a partir dos 15 anos.
Um grande número dos jovens nasceu na Região Metropolitana de São Paulo $(82,8 \%$ ou 336), apesar do fato de que uma considerável proporção de seus pais e mães (69,5\% e 68,0\% respectivamente) nasceu em outras regiões do país, principalmente o Nordeste, e migrado para a cidade de São Paulo.

Neste presente estudo, foram considerados apenas os jovens que já haviam iniciado sua vida sexual, ou seja, quase metade dos adolescentes $(46,1 \%$ ou 187$)$, sendo que 86 eram do sexo masculino $(45,9 \%)$ e 101 eram do sexo feminino $(54,1 \%)$.

Foram estudadas as seguintes variáveis, sempre segundo o sexo: idade média do início da vida sexual (anos); tipo de relacionamento com o primeiro parceiro sexual (estável, considerando as relações de namoro e noivado e não estável, considerando os outros tipos de relacionamento); idade média do primeiro parceiro sexual (anos); local da ocorrência da primeira relação sexual (em casa e fora de casa); planejamento prévio da primeira relação sexual (sim e não); uso de algum tipo de método contraceptivo na primeira e na última relação sexual (sim e não); tipo de método contraceptivo utilizado na primeira e na última relação sexual (preservativo masculino e outros); número médio de parceiros sexuais. A análise dos fatores associados ao sexo foi realizada por meio do teste de associação pelo Qui-quadrado, com correção de Yates. Os valores médios encontrados nas variáveis quantitativas contínuas foram comparados pelo teste de Mann-Whitney.

\section{Resultados}

A primeira relação sexual ocorreu em média aos 15,13 anos de idade. Não foi observada diferença estatisticamente significativa entre a idade média de início da vida sexual entre homens e mulheres $(14,94$ e 15,29 anos respectivamente). Os adolescentes de ambos os sexos iniciaram sua vida sexual com parceiros(as) mais velhos(as), porém cabe ressaltar que a diferença de idade entre o adolescente e seu primeiro parceiro foi bem maior entre as mulheres do que os homens (Tabela 1).

As mulheres relataram ter iniciado sua vida sexual predominantemente com pessoas com quem já tinham um relacionamento afetivoamoroso estável, como em um namoro $(79,1 \%)$ ou noivado $(7,0 \%)$, mas este evento foi também observado em relacionamentos de amizade $(9,9 \%)$ ou com uma pessoa recém-conhecida $(4,0 \%)$. Por outro lado, os homens iniciaram sua vida sexual em relações de amizade $(47,7 \%)$, 


\section{Tabela 1}

Média, mediana, desvio-padrão, valor máximo e mínimo da idade (em anos) do início da vida sexual dos adolescentes entrevistados e de seus primeiros parceiros sexuais, por sexo. São Paulo, Brasil, 2002

\begin{tabular}{lrrr}
\hline \multirow{2}{*}{ Variável (anos) } & \multicolumn{2}{c}{ Sexo } & Média geral \\
& Masculino & Feminino & \\
\hline Idade na primeira relação sexual & & & \\
$\quad$ Média & 14,94 & 15,28 & 15,13 \\
Mediana & 15,00 & 15,00 & 15,00 \\
Desvio-padrão & 1,53 & 1,40 & 1,47 \\
Valor máximo-mínimo & $8-18$ & $11-18$ & $8-18$ \\
& & & \\
Idade do(a) primeiro(a) parceiro sexual* & 16,36 & 19,58 & 18,10 \\
Média & 16,00 & 19,00 & 17,00 \\
Mediana & 3,08 & 3,47 & 3,66 \\
Desvio-padrão & $8-28$ & $14-30$ & $8-30$ \\
Valor máximo-mínimo & & & \\
\hline
\end{tabular}

* $\mathrm{p}<0,01$ planejamento desta primeira relação, como por exemplo, "não esperava ter relações naquele dia" (36,4\%), "não tinha preservativo no momento" $(16,9 \%)$ e "nem pensei sobre isto" (14,3\%), entre outros. Não houve diferença estatisticamente significativa quanto ao uso de contraceptivos na primeira relação sexual entre homens e mulheres (Tabela 2).

Com respeito à última relação sexual, observou-se que houve um aumento da proporção dos jovens que utilizaram algum método anticonceptivo em comparação com a primeira relação sexual, contudo, este incremento não se mostrou homogêneo em ambos os sexos (Tabela 2). O tipo de método utilizado foi também diferenciado. Verificou-se que a proporção do uso de preservativo masculino entre os homens permaneceu alta, ao passo que, entre as mulheres, o condom foi parcialmente substituído pela pílula ou outros métodos hormonais (Tabela 3).

Os homens revelaram ter tido 5,1 parceiros(as) sexuais até o momento da entrevista, enquanto as mulheres referiram ter tido um pouco menos da metade, média de 2,1 parcei$\operatorname{ros}(\mathrm{as})(\mathrm{p}<0,01)$. meira relação sexual com um(a) namorado(a) ocorreu com $32,6 \%$ dos homens.

Dentro deste contexto, foi observada uma diferença estatisticamente significativa entre homens e mulheres em relação ao tipo de relacionamento com o(a) primeiro(a) parceiro(a). Considerado aqui o namoro o relacionamento afetivo-amoroso estável mais esperado e freqüente da adolescência, pode-se dizer que 87 mulheres $(86,2 \%)$ iniciaram sua vida sexual em um relacionamento estável (compreendendo também o noivado), ao passo que apenas 28 $(32,6 \%)$ homens o fizeram (Tabela 2). Alguns adolescentes não mantiveram qualquer tipo de relacionamento afetivo com o primeiro parceiro sexual posteriormente à primeira relação sexual, compreendendo $29,0 \%$ dos homens e $4,0 \%$ das mulheres.

A primeira relação sexual foi referida como tendo "simplesmente acontecido" por $72,7 \%$ dos adolescentes. O não planejamento da primeira relação sexual foi semelhante em ambos os sexos, assim como sua ocorrência com maior freqüência dentro de casa, seja a do próprio adolescente, a do parceiro ou a de parentes (Tabela 2).

Em relação ao uso de métodos contraceptivos, 114 jovens $(61,0 \%)$ relataram ter utilizado algum método na primeira relação sexual, majoritariamente o preservativo masculino (96,5\%). Dentre os que não utilizaram o preservativo masculino na primeira relação sexual, as principais razões alegadas dizem respeito ao não

\section{Discussão}

A tendência de antecipação da idade do início da vida sexual mais intensamente entre as jovens do sexo feminino foi confirmada comparando com os resultados apontados em outros estudos de âmbito nacional 4,5 . Neste presente estudo, não somente as adolescentes iniciaram sua vida sexual mais precocemente se comparadas a outras gerações, mas tiveram a primeira relação sexual praticamente com a mesma idade que os homens (mediana de 15 anos), ao contrário dos resultados encontrados por BEMFAM 6, Pirotta 7, Almeida et al. 13 e Aquino et al. 14. No entanto, é preciso considerar que estudos conduzidos com populações jovens da Suécia, dos Estados Unidos e do Reino Unido, têm observado que a idade média na primeira relação sexual das mulheres vem se mantendo similar ao longo dos últimos anos, com uma particular convergência desta idade entre homens e mulheres $15,16,17$, sugerindo que a progressiva diminuição da idade de iniciação sexual alcança um certo limite e passa, a partir de então, a se manter em níveis estáveis, fenômeno que pode vir a ocorrer em futuro próximo também no Brasil.

A iniciação sexual de garotas e garotos em uma mesma idade é um fato interessante por ocorrer em uma cultura que tradicionalmente 
Distribuição por sexo segundo o tipo de relacionamento com o(a) primeiro(a) parceiro(a) sexual, planejamento e local da primeira relação sexual, uso de métodos contraceptivos (MAC) na primeira e última relação sexual. São Paulo, Brasil, 2002.

\begin{tabular}{|c|c|c|c|c|c|c|}
\hline \multirow[t]{3}{*}{ Variável } & \multicolumn{4}{|c|}{ Sexo } & \multicolumn{2}{|c|}{ Total } \\
\hline & \multicolumn{2}{|c|}{ Masculino } & \multicolumn{2}{|c|}{ Feminino } & \multirow[b]{2}{*}{$\mathrm{n}$} & \multirow[b]{2}{*}{$\%$} \\
\hline & $\mathrm{n}$ & $\%$ & $\mathrm{n}$ & $\%$ & & \\
\hline \multicolumn{7}{|c|}{ Relacionamento com o 1ㅇ parceiro sexual* } \\
\hline Estável & 28 & 32,5 & 87 & 86,2 & 115 & 61,5 \\
\hline Não-estável & 58 & 67,5 & 14 & 13,8 & 72 & 38,5 \\
\hline \multicolumn{7}{|c|}{ Planejamento da 1 a relação sexual } \\
\hline Não & 67 & 73,0 & 69 & 68,4 & 136 & 72,7 \\
\hline Sim & 19 & 22,0 & 32 & 31,6 & 51 & 27,3 \\
\hline \multicolumn{7}{|c|}{ Local da 1ạ relação sexual } \\
\hline Em casa & 70 & 81,4 & 91 & 90,1 & 161 & 86,1 \\
\hline Fora de casa & 16 & 18,6 & 10 & 9,9 & 26 & 13,9 \\
\hline \multicolumn{7}{|c|}{ Uso MAC na 1ạ relação sexual } \\
\hline Não & 35 & 40,7 & 38 & 37,6 & 73 & 39,0 \\
\hline Sim & 51 & 59,3 & 63 & 62,4 & 114 & 61,0 \\
\hline \multicolumn{7}{|c|}{ Uso MAC na última relação sexual** } \\
\hline Não & 15 & 17,4 & 31 & 30,7 & 46 & 24,6 \\
\hline Sim & 71 & 82,6 & 70 & 69,3 & 141 & 75,4 \\
\hline Total & 86 & 100,0 & 101 & 100,0 & 187 & 100,0 \\
\hline
\end{tabular}

* $p<0,01$;

$\star \star p<0,05$

Tabela 3

Distribuição por sexo segundo o tipo de método contraceptivo usado na primeira e na última relação sexual. São Paulo, Brasil, 2002.

\begin{tabular}{|c|c|c|c|c|c|c|}
\hline \multirow[t]{3}{*}{ Tipo de contraceptivos utilizados } & \multicolumn{4}{|c|}{ Sexo } & \multicolumn{2}{|c|}{ Total } \\
\hline & \multicolumn{2}{|c|}{ Masculino } & \multicolumn{2}{|c|}{ Feminino } & \multirow[b]{2}{*}{$\mathrm{n}$} & \multirow[b]{2}{*}{$\%$} \\
\hline & $\mathrm{n}$ & $\%$ & $\mathrm{n}$ & $\%$ & & \\
\hline \multicolumn{7}{|l|}{ Primeira relação sexual $(n=114)$} \\
\hline Preservativo masculino & 51 & 100,0 & 59 & 93,7 & 110 & 96,5 \\
\hline Outros & - & - & 4 & 6,3 & 4 & 3,5 \\
\hline \multicolumn{7}{|l|}{ Última relação sexual $(n=141)^{\star}$} \\
\hline Preservativo masculino & 69 & 97,2 & 46 & 65,7 & 115 & 81,6 \\
\hline Outros & 2 & 2,8 & 24 & 34,3 & 26 & 18,4 \\
\hline
\end{tabular}

${ }^{\star} p<0,01$. 
tem estimulado os jovens do sexo masculino a iniciarem suas práticas sexuais bem mais cedo do que o sexo feminino. Assim, este fenômeno recentemente observado pode ser uma evidência das transformações ocorridas no comportamento sexual da população brasileira, por conta da entrada maciça das mulheres no mercado de trabalho e sua crescente escolarização, do uso generalizado de métodos contraceptivos modernos, permitindo a desvinculação do ato sexual à reprodução e, principalmente no contexto da AIDS, no qual as questões em torno da sexualidade tornaram-se mais proeminentes no cenário público brasileiro 18 .

Considerando a idade média dos primeiros parceiros sexuais, os dados mostraram que os jovens tiveram a primeira relação sexual com pessoas mais velhas. É preciso observar que os primeiros parceiros sexuais das mulheres, sendo quase quatro anos mais velhos que elas, podem acarretar perdas no poder de negociação e autonomia de decisão tanto em relação ao momento de iniciar a vida sexual quanto na escolha do uso e tipo de métodos anticonceptivos pelas adolescentes, coerentemente com o que sugeriu Longo 19. Este resultado também reforça o tradicional relacionamento de mulheres brasileiras com parceiros mais velhos 20 e, por conseqüência, mais experientes sexualmente e, provavelmente, mais expostos aos riscos de contrair DST/AIDS.

Se a idade em que a primeira relação sexual das mulheres ocorreu foi antecipada e igualada à dos homens, não se pode dizer o mesmo dos contextos em que se deu esta primeira relação sexual. As mulheres continuaram, em sua maioria $(82,9 \%)$, guardando a primeira vez para ser compartilhada com pessoas cujo relacionamento compreendesse um compromisso, como, por exemplo, namorados ou noivos, persistindo uma idéia não apenas romântica sobre o sexo, mas também de "entrega”. Pantoja 21 (p. 339), por meio de um estudo antropológico com meninas adolescentes em Belém, Pará, Brasil, descreveu que "no relato das mesmas, a primeira experiência sexual aparece como formulada em termos de uma 'entrega', cuja legitimidade ocorre no âmbito de uma relação afetiva já consolidada (o namoro), concretizada a partir da apresentação do parceiro à família, seguida do 'pedido' em namoro".

Heilborn 18 (p. 56) enfatizou, ainda, as diferenças marcantes no início da vida sexual entre homens e mulheres: " $a$ entrada na vida sexual adulta e a maneira como as mulheres vivem esta passagem continuam a diferir fortemente daquelas dos homens; enquanto para elas a primeira relação sexual é freqüentemente um mo- mento decisivo (e inicial) na construção do primeiro relacionamento verdadeiro, para eles trata-se de um momento de iniciação pessoal no qual a relação com a parceira conta pouco". E acrescentou, juntamente com Bozon 22 (p. 130) que, para os homens, "a primeira relação tem um caráter de prova, de experiência aventureira e arriscada. Os discursos masculinos são centrados sobre o indivíduo, sua satisfação ou suas dúvidas sobre si mesmo, a parceira não é o foco de interesse".

No tocante aos homens, a maior parte de suas primeiras experiências sexuais ocorreu em relacionamentos não estáveis. No entanto, uma proporção considerável dos homens também relacionou-se sexualmente pela primeira vez com namorados(as), compreendendo $32,6 \%$. A este respeito, Rieth 23 (p. 128) descreveu que "a experiência masculina (...) traduz-se em duas atitudes: numa, o desempenho sexual é visto como um ganho, sustentando o poder da masculinidade, noutra, a atitude é decididamente romântica, em que o homem busca 'entregar-se' no momento certo e à parceira certa". Neste contexto, Pirotta 7 constatou um forte desejo masculino de iniciar a vida sexual em um relacionamento com vínculo afetivo-amoroso, o que pode sugerir ser esta uma tendência crescente entre os adolescentes do sexo masculino.

A primeira relação sexual não foi planejada pela maior parte dos jovens, sem diferença entre homens e mulheres. Ao também considerar o local em que esta primeira relação sexual ocorreu, majoritariamente dentro de casa, pode-se aferir que houve um certo improviso e até mesmo pressa em terminar o ato sexual, visto que os jovens, além de lidarem com todas as ansiedades e preocupações que normalmente permeiam o início da vida sexual, tiveram também como preocupação a eminência da chegada de algum membro da família que pudesse surpreender este momento, fazendo com que, possivelmente, outras prioridades fossem colocadas em primeiro plano em detrimento de atitudes voltadas à contracepção e prevenção de DST/AIDS.

A prática contraceptiva foi deixada de lado por cerca de metade dos jovens, fossem homens ou mulheres. Os resultados demonstraram que muitos jovens ainda estão iniciando sua vida sexual sem proteção contra gestações não planejadas e DST/AIDS, em concordância com outros estudos 13,19.

Apesar do conhecimento de adolescentes sobre os métodos contraceptivos ser considerado praticamente universal $6,24,25$, pelo menos em relação à pílula, e gradativamente, em relação ao condom, Vieira 26 (p. 174) salientou que 
não há, necessariamente, um conhecimento qualitativamente adequado, o que "facilita o uso inadequado, aumenta os índices de falha, criando situações nas quais mitos sobre anticoncepcionais são construídos". Neste sentido, cabe relatar que, tanto Romer et al. 27 quanto Almeida et al. 13, não encontraram associação entre o nível de conhecimento acerca dos métodos contraceptivos e seu uso entre os adolescentes, revelando que outros fatores fazem-se mais presentes na determinação do uso da anticoncepção na primeira e nas relações sexuais posteriores.

Entre todos os jovens, homens ou mulheres, que relataram não ter utilizado o preservativo masculino na primeira vez, a maioria respondeu que não houve preparo prévio, do ponto de vista contraceptivo, para a primeira relação sexual, sugerindo, mais uma vez, um início da vida sexual improvisado, evidenciado a partir de respostas como, por exemplo, o fato de não possuir o condom no momento do ato sexual ou não esperar ter relações sexuais naquele dia.

Os resultados mostraram que o uso do preservativo masculino foi mais freqüente entre os homens, semelhante ao estudo realizado por Almeida et al. 13, onde encontraram uma proporção de uso do condom na primeira relação sexual na ordem de $90,1 \%$ e $73,5 \%$, entre homens e mulheres, respectivamente. Para Camarano 1 , o condom foi o método anticonceptivo mais referido pelos homens que tinham entre 15 e 19 anos de idade em 1996 e que usavam algum tipo de método, refletindo, provavelmente, uma maior preocupação com as DST/AIDS do que com a gravidez propriamente dita.

O percentual de jovens que utilizou algum método contraceptivo na última relação sexual foi maior quando comparado ao percentual de jovens que utilizou na primeira relação sexual. Entretanto, o aumento foi maior entre os homens, que relataram, novamente, o uso predominante do preservativo masculino.

As adolescentes do sexo feminino também ampliaram o uso de anticonceptivos na última relação sexual, porém os resultados mostraram que o preservativo masculino foi parcialmente substituído por outros métodos, tais como o contraceptivo hormonal oral ou injetável. Isto pode ser explicado, mais enfaticamente, pelo fato de que, apesar de as adolescentes entrevistadas terem iniciado sua vida sexual fora de uma união e em idade mais precoce, elas substituíram o condom como método contraceptivo de escolha justamente por estarem inseridas em um relacionamento mais estável e com vínculo afetivo-amoroso, ratificando uma tendência de abandono do preservativo masculino na vigência da estabilização do relacionamento, como prova de amor, confiança e fidelidade ao parceiro ou como conseqüência de uma sensação de proteção e imunidade ligados ao sentimento de amor, conforme já descreveram Paiva 28, Pirotta 7 e Antunes et al. 29.

Pirotta 7 discutiu que o uso do condom está associado a determinadas situações na vida dos jovens, como a primeira relação sexual ou relações sexuais esporádicas com parceiros pouco conhecidos, além do início de um relacionamento com novos parceiros, o que pode explicar a alta proporção de uso deste método entre os rapazes, que haviam trocado mais de parceiros(as) sexuais até aquele momento do que as mulheres e, por isto, mostraram-se menos suscetíveis às conseqüências de uma relação sexual desprotegida do que as adolescentes do sexo feminino. Este fenômeno pode ser considerado um dos determinantes da inversão da razão de casos de AIDS entre homens e mulheres de 13 a 19 anos de idade no período de 1998 a 2001, visto que há o predomínio da categoria transmissão sexual entre as mulheres 2 .

Concluindo, os resultados evidenciaram que, mesmo que a primeira relação sexual esteja ocorrendo em idades muito próximas entre homens e mulheres, aspectos importantes do comportamento sexual diferem entre os adolescentes de ambos os sexos e devem ser levados em consideração na assistência e promoção de sua saúde reprodutiva. As questões de gênero, ao sugerirem condutas diferenciadas para homens e mulheres em relação à primeira relação sexual, ao tipo de vínculo com o primeiro parceiro e às decisões acerca das práticas contraceptivas, geram impacto importante no processo de decisão de relacionar-se sexualmente de formas mais ou menos seguras e necessitam ganhar espaço na elaboração das políticas públicas voltadas ao adolescente. 


\section{Resumo}

Trata-se de estudo transversal com o objetivo de identificar os diferenciais de gênero presentes no início da vida sexual de adolescentes. Foram entrevistados 406 adolescentes de 15 a 19 anos de idade inscritos em uma unidade de saúde da família da zona leste do Município de São Paulo, Brasil, entre maio e dezembro de 2002. Quase a metade dos adolescentes já havia iniciado sua vida sexual, em média aos 15 anos de idade, fossem homens ou mulheres. O início da vida sexual ocorreu, em grande parte, de forma não planejada $(72,7 \%)$ e dentro de casa $(86,1 \%)$. O uso de um método contraceptivo na primeira relação sexual foi similar entre homens e mulheres $(61,0 \%)$, entretanto, foi observado um aumento maior na proporção de uso de algum anticonceptivo entre os homens na última relação sexual. Pelo fato de terem iniciado sua vida sexual em relacionamentos estáveis com vínculos afetivoamorosos, as mulheres mostraram-se mais vulneráveis às DST/AIDS, pois substituíram o preservativo masculino por outros métodos na última relação sexual, enquanto os homens permaneceram utilizando largamente o condom, provavelmente por estarem inseridos em relacionamentos não estáveis.

Saúde Reprodutiva; Adolescente; Relações Interpessoais

\section{Colaboradores}

A. L. V. Borges participou de todas as etapas do estudo e da elaboração do artigo, sendo responsável pela versão final. N. Schor participou de todas as etapas do estudo e da elaboração do artigo.

\section{Referências}

1. Camarano AA. Fecundidade e anticoncepção da população jovem. In: Comissão Nacional de População e Desenvolvimento, organizador. Jovens acontecendo na trilha de políticas públicas. Brasília: Comissão Nacional de População e Desenvolvimento; 1998. p. 109-33.

2. Ministério da Saúde. Boletim epidemiológico AIDS 2002; ano XVI, n. 1.

3. Ferraz E, Ferreira IQ. Início da atividade sexual e características da população adolescente que engravida. In: Vieira EM, Fernandes MEL, Bailey P, McKay A, organizadores. Seminário gravidez na adolescência. Rio de Janeiro: Associação Saúde da Família; 1998. p. 47-54.

4. Ministério da Saúde. Pesquisa sobre comportamento sexual e percepções da população brasileira sobre HIV/AIDS. Brasília: Coordenação Nacional de DST e AIDS; 2000.

5. Melo AV, Yazaki LM. O despertar do desejo. In: Secretaria de Economia e Planejamento, Fundação Sistema Estadual de Análise de Dados, organizador. 20 anos no ano 2000: estudos sócio-demográficos sobre a juventude. São Paulo: Fundação Sistema Estadual de Análise de Dados; 1998. p. 119-25.

6. Sociedade Civil Bem-Estar Familiar no Brasil/ Programa de Pesquisas de Demografia e Saúde Macro International. Brasil - Pesquisa Nacional sobre Demografia e Saúde - 1996. Rio de Janeiro: Sociedade Civil Bem-Estar Familiar no Brasil; 1997.

7. Pirotta KCM. Não há guarda-chuva contra o amor: estudo do comportamento reprodutivo e de seu universo simbólico entre jovens universitários da USP [Tese de Doutorado]. São Paulo: Faculdade de Saúde Pública, Universidade de São Paulo; 2002.

8. Monteiro S. Gênero, sexualidade e juventude numa favela carioca. In: Heilborn ML, organizador. Sexualidade: o olhar das ciências sociais. Rio de Janeiro: Jorge Zahar Editor; 1999. p. 117-45.

9. Heilborn ML. A primeira vez nunca se esquece. Revista Estudos Feministas 1998; 6:394-405.

10. Instituto Brasileiro de Geografia e Estatística. Censo demográfico 2000. Características gerais da população: resultados da amostra. Rio de Janeiro: Instituto Brasileiro de Geografia e Estatística; 2003.

11. Pinho MD, Berquó E, Oliveira KA, Lopes F, Lima LCA, Pereira N. Juventudes, raça e vulnerabilidades. Revista Brasileira de Estudos Populacionais 2002; 19:277-94.

12. Carvalho SN, Belluzzo L, Ferreira MP, Nery EG. Situação educacional e condições sociais. In: Secretaria de Economia e Planejamento, Fundação Sistema Estadual de Análise de Dados, organizador. 20 anos no ano 2000: estudos sócio-demográficos sobre a juventude. São Paulo: Fundação Sistema Estadual de Análise de Dados; 1998. p. 186-94.

13. Almeida MCC, Aquino EML, Gaffikin L, Magnani RJ. Uso de contracepção por adolescentes de escolas públicas na Bahia. Rev Saúde Pública 2003; 37:566-75.

14. Aquino EML, Heilborn ML, Knauth D, Bozon M, Almeida MC, Araújo J, et al. Adolescência e repro- 
dução no Brasil: a heterogeneidade dos perfis sociais. Cad Saúde Pública 2003; 19 Suppl 2:377-88.

15. Singh S, Darroch JE. Trends in sexual activity among adolescent American women 1982-1995. Fam Plann Perspect 1999; 31:212-9.

16. Wellings $\mathrm{K}$, Nanchahal $\mathrm{K}$, MacDowall W, McManus S, Erens B, Mercer CH, et al. Sexual behaviour in Britain: early heterosexual experience. Lancet 2001; 358:1843-50.

17. Häggström-Nordin E, Hanson U, Tydén T. Sex behavior among high school students in Sweden: improvement in contracpetive use over time. J Adolesc Health 2002; 30:288-95.

18. Heilborn ML. Construção de si, gênero e sexualidade. In: Heilborn ML, organizador. Sexualidade: o olhar das ciências sociais. Rio de Janeiro: Jorge Zahar Editor; 1999. p. 40-58.

19. Longo LAFB. Juventude e contracepção: um estudo dos fatores que influenciam o comportamento contraceptivo das jovens brasileiras de 15 a 24 anos. Revista Brasileira de Estudos Populacionais 2002; 19:229-47.

20. Berquó E. Quando, como e com quem se casam os jovens brasileiros. In: Comissão Nacional de População e Desenvolvimento, organizador. Jovens acontecendo na trilha de políticas públicas. Brasília: Comissão Nacional de População e Desenvolvimento; 1998. p. 93-107.

21. Pantoja ALN. "Ser alguém na vida": uma análise sócio-antropológica da gravidez/maternidade na adolescência, em Belém do Pará, Brasil. Cad Saúde Pública 2003; 19 Suppl 2:335-43.

22. Bozon M, Heilborn ML. As carícias e as palavras: iniciação sexual no Rio de Janeiro e em Paris. Novos Estudos CEBRAP 2001; 59:111-35.
23. Rieth F. Ficar e namorar. In: Bruschini C, Hollanda HB, organizadores. Horizontes plurais: novos estudos de gênero no Brasil. São Paulo: Editora 34; 1998. p. 113-33.

24. Schor N. Adolescência e anticoncepção: conhecimento e uso [Tese de Livre-Docência]. São Paulo: Faculdade de Saúde Pública, Universidade de São Paulo; 1995.

25. Morell MGG, Yazaki LM. Anticoncepção e preferências reprodutivas. In: Secretaria de Economia e Planejamento, Fundação Sistema Estadual de Análise de Dados, organizador. 20 anos no ano 2000: estudos sócio-demográficos sobre a juventude. São Paulo: Fundação Sistema Estadual de Análise de Dados; 1998. p. 136-48.

26. Vieira EM. Políticas públicas e contracepção no Brasil. In: Berquó E, organizador. Sexo e vida: panorama da saúde reprodutiva no Brasil. Campinas: Editora Unicamp; 2003. p. 151-96.

27. Romer D, Black M, Ricardo I, Feigelman S, Kaljee L, Galbraith J, et al. Social influences on the sexual behavior of youth at risk of HIV exposure. Am J Public Health 1994; 84:977-85.

28. Paiva V. Fazendo arte com camisinha. Sexualidades em tempos de Aids. São Paulo: Summus; 2000.

29. Antunes MC, Peres CA, Paiva V, Stall R, Hearst N. Diferenças na prevenção da Aids entre homens e mulheres jovens de escolas públicas em São Paulo, SP. Rev Saúde Pública 2002; 36 (4 Suppl):88-95.

Recebido em 28/Nov/2003

Versão final reapresentada em 30/Ago/2004

Aprovado em 20/Set/2004 